Journal of Bangladesh Academy of Sciences, Vol. 36, No. 1, 33-37, 2012

\title{
ON SOME CHARACTERIZATIONS OF SURFACES WITH CONSTANT CURVATURE ZERO
}

\author{
MOHD. ALTAB HOSSAIN* \\ Department of Mathematics, University of Rajshahi, Rajshahi-6205, Bangladesh
}

\begin{abstract}
A class of surfaces with constant vanishing curvature is studied in this paper. The importance of this study lies in the fact that some of the special structures of this class of surfaces have been completely established.
\end{abstract}

Key words : Constant curvature, Planar point, Ruled surface, Developable surface, Isometric mapping, Local structure

\section{INTRODUCTION}

Since study of surfaces is an important part of differential geometry it has been extensively studied from various perspectives. Authors (Kreyszig 1991, Lopez 2001, Pressley 2001) studied a number of classes of surfaces. In some particular situations, a very few of these surfaces are of constant curvature $K$ ( $\mathrm{K}$ being greater than zero, equal to zero and less than zero). For examples, the simply connected surfaces of constant curvature 0 , +1 and -1 are the Euclidean plane, the unit sphere in $\mathbf{E}^{3}$, and the hyperbolic plane, respectively. In the case when $K=0$ on a surface, then the surface belongs to a particular class and it is called the surface with vanishing curvature or, zero curvature. If the curvature $K=0$ everywhere on a surface, then it called locally flat surface also. Also, the point $P \in S$ where $K>0, K<0$ and $K=0$ are characterized by the elliptic point, the hyperbolic point and the parabolic point respectively. Particularly, Castro and Montealegre (2004) and Hossain (2010) studied on some nature of surfaces with specific constant curvature, whereas Kenmotsu (1983) dealt with the study of minimal surfaces of constant curvature in relatively high dimensional space. This paper deals with the study of surfaces of exactly zero curvature only. For convenience, some terminologies and notions of Castro and Montealegre (2004) and Kreyszig (1991) have been used frequently here.

\section{NECESSARY PRELIMINARIES}

Let $S$ be an embedded surface and let every $x^{i}(i=1,2,3)$ be the Cartesian coordinates of any $p \in S$. Then there are parameterizations $\underline{x}=\underline{x}\left(u^{\alpha}\right) ; \alpha=1,2$, having $u^{1}=u^{1}, u^{2}=$ const. and $u^{1}=$ const.,$u^{2}=u^{2}$ as associated families of paths called the

\footnotetext{
*Corresponding author: <al_math_bd@yahoo.com>
} 
parametric net of the parameterization. Note that over the whole of $S, g$ defines a differentiable tensor field with components $g_{11}=E, g_{12}=g_{21}=F, g_{22}=G$. This $g$ is called the $1^{\text {st }}$ fundamental tensor or metric tensor where $E, F, G$ are the $1^{\text {st }}$ fundamental coefficients. Now for $\underline{v}, \underline{w} \in T_{p}(S)$ if we set $\in(\underline{v}, \underline{w})=(\underline{N}, \underline{v}, \underline{w})$, there corresponds an anti-symmetric tensor with components given by $\epsilon_{\alpha \beta}=\in\left(\underline{x_{\alpha}}, \underline{x_{\beta}}\right)=\left(\underline{N}, \underline{x}_{\alpha}, \underline{x}_{\beta}\right) ; \underline{N}$ being the unit normal to $S$. Since $\underline{x_{\alpha}}$ are tangents to $S$, we can take $\underline{N}=\frac{\left(x_{1} \times \underline{x_{2}}\right)}{1}$, where $(\operatorname{det} g)^{\frac{1}{2}}$

$\operatorname{det} g=\operatorname{det}\left(g_{\alpha \beta}\right)=g_{12} g_{22}-g_{12}{ }^{2}$. Thus $\epsilon_{11}=\epsilon_{22}=0, \epsilon_{12}=-\epsilon_{21}=[\operatorname{det}(g)]^{\frac{1}{2}}$. Define a natural mapping $\gamma: S \rightarrow S^{2}, S^{2}$ being the unit 2-sphere, by sending the point $p \in S$ to the point with radius vector $\underline{N}(p)$ of $S^{2}$. Then $\gamma$ is called the Gauss map or the spherical image of $S$, and the linear map $l=-\gamma_{p}^{\prime}: T_{p}(S) \rightarrow T_{p}(S)$ is known as the Weingarten transformation. Since $\underline{N}_{\alpha} \in T_{p}(S)$, there are some coefficients $b_{\alpha}^{\beta}$ such that $\underline{N}_{\alpha}=-b_{\alpha}^{\beta} \underline{x}_{\beta}$ which are known as the Weingarten formulas. It is true that the Weingarten transformation is self-adjoint. Thus if we define $b(\underline{v}, \underline{w})=l \underline{v} \cdot \underline{w}=\underline{v} \cdot l \underline{w}$, there corresponds a symmetric bilinear map $b: T_{p}(S) \times T_{p}(S) \rightarrow \boldsymbol{R}$, hence a symmetric two-times covariant tensor at $p \in S$ with components given by

$$
b_{\alpha \beta}=b\left(\underline{x}_{\alpha}, \underline{x}_{\beta}\right)=l \underline{x}_{\alpha} \cdot \underline{x}_{\beta}=-\underline{N}_{\alpha} \cdot \underline{x}_{\beta}=-\underline{N}_{\beta} \cdot \underline{x}_{\alpha}=\underline{N} \cdot \underline{x}_{\alpha \beta} .
$$

If $p$ varies on $S, b$ defines a tensor field which will be referred to as the $2^{\text {nd }}$ fundamental tensor or form of $S$. It can be shown that $b_{11}=L, b_{12}=b_{21}=M, b_{22}=N$, where L, M, N are the $2^{\text {nd }}$ fundamental coefficients. If $\underline{v}$ is an eigenvector of the Weingarten transformation $l$ i.e., some scalar $k$ exists such that $l(\underline{v})=k \underline{v}$, then the corresponding eigenvalues $k$ of $l$ are called the principal curvature. Due to the above definitions, one can easily derive the following equation involving the principal curvature $k$ :

$$
\operatorname{det}\left(b_{\gamma \beta}-k g_{\gamma \beta}\right)=0 \text { yielding }\left|\begin{array}{ll}
b_{11}-k g_{11} & b_{12}-k g_{12} \\
b_{21}-k g_{21} & b_{22}-k g_{22}
\end{array}\right|=0 \text { which is of the second }
$$

degree in $k$. If $k_{1}, k_{2}$ are the two values of $k$, then (i) $H=k_{1}+k_{2}$ is called the mean curvature of $S$ at $p \in S$, and (ii) $K=k_{1} k_{2}$ is called the total curvature or Gaussian curvature of $S$ at $p \in S$. In this situation, one must have $k^{2}-H k+K=0$, $H=g^{\alpha \beta} b_{\alpha \beta}=\operatorname{tace}(l)$, and $K=\frac{\operatorname{det}\left(b_{\alpha \beta}\right)}{\operatorname{det}\left(g_{\alpha \beta}\right)}=\operatorname{det}(l)$. These are the very important invariants of a surface. Sometimes, $K$ is constant throughout the surface then the surface is known as that with constant curvature.

A surface generated by one-parameter family of straight lines is called a ruled surface. If $y(\tau), \tau$ being a parameter, is a curve that intersects the generators of the ruled 
surface, and if $\underline{v}(\tau)$ defines the direction of the generator through $\underline{y}(\tau)$, the equation of the generator will be of the form $\underline{x}=\underline{y}(\tau)+\lambda \underline{v}(\tau)$, where $\tau, \lambda$ are two independent parameters. Therefore, one can think that a ruled surface $S$ has a regular parameterization of the form $\underline{x}=y\left(u^{1}\right)+u^{2} \underline{v}\left(u^{1}\right) \quad \cdots \cdots(*)$.

Theorem 2.1: The curvature of a surface depends only on the first fundamental form of the surface.

A point $p$ on a regular surface $S$ is said to be planar point if the Gaussian curvature $K(p)=0$ and $s(p)$ (where $\mathrm{s}$ is the shape operator), or equivalently, both of the principal curvatures $k$ and $k_{2}$ are 0 .

Proposition 2.2: If an open connected subset $U$ of a surface $S$ consists of planar points only, then $U$ belongs to a plane.

\section{SRUCTURES OF THE CONSIDERED CLASS OF SURFACES}

It is more difficult to establish the global structure of the class of surfaces under investigation. If the surface is required to be complete, one can show that it must be either a plane or a cylinder. Some of the required structures have made locally also. The geometric structures of the surfaces with curvature zero have determined through the last paragraph of these section.

Theorem 3.1: Let $S$ be a surface of curvature zero with no planar points. Then $S$ must be a ruled surface whose tangent plane is constant along every generator.

Proof: Consider a surface $S$ with constant curvature zero and no planar points. Suppose that $p \in S$ is not a planar point. Since $K(p)=k_{1}(p) \cdot k_{2}(p)=0$, we must have, for example, $k_{1}=0, k_{2} \neq 0$. Then $p$ is not umbilical, and there is a parameterization $\underline{x}\left(u^{1}, u^{2}\right)$ at $p$ so that the $u^{1}$-curves are the 1 st lines of curvatures and the $u^{2}$-curves are the 2 nd lines of curvatures i.e., the parameterization $x\left(u^{1}, u^{2}\right)$ has the lines of curvature as its parametric net. Moreover, it may be assumed that $k_{1}=0$ along the lines $u^{1}=$ const. and that $u^{2}$ is the arc length along $u^{1}=0$. If this is not so from the beginning, we replace the parameters by $v^{1}=u^{1}, v^{2}=\int\left[g_{22}(0, u)\right]^{\frac{1}{2}} d u^{2}$. Now using the Weingarten transformation, we obtain $N_{1}=-k_{2} x_{1}, \quad N_{2}=0$ and since $g_{12}=0$ and $b_{12}=0$, so $x_{1} \cdot x_{2}=0, N_{1} \cdot x_{2}=0$. Since $N \cdot x_{2}=0$ and $N_{2}=0$, we have by a differentiation $\underline{N} \cdot \underline{x_{22}}=0 . \bar{A}$ gain, differentiating $\quad N_{1} \cdot x_{2}=\overline{0}$ with respect to $u^{2}$ and using $\underline{N}_{22}=\underline{N}_{21}=0$ and $\underline{N}_{1}=-k_{2} \underline{x_{1}}\left(k_{2} \neq \overline{0)}\right.$, we get $\underline{x_{1}} \cdot \underline{x_{22}}=0$.

Now $\partial\left(\underline{\left.x_{2}{ }^{2}\right)}\right) / \partial u^{1}=2 \underline{x_{2}} \cdot \underline{x_{21}}=-2 \underline{x_{1}} \cdot \underline{x_{22}}$. Hence $\partial\left(\underline{\left.x_{2}{ }^{2}\right)} / \partial u^{1}=0\right.$, and $\underline{x}_{2}{ }^{2}$ does not depend $u^{1}$. Since $u^{2}$ is the arc length $\overline{\text { on }} u^{1}=0$ we have $x_{2}{ }^{2}\left(0, u^{2}\right)=1$ and, therefore $\underline{x_{2}}{ }^{2}\left(u^{1}, u^{2}\right)=1$. By a differentiation, this yields $\underline{x_{2}} \cdot \underline{x_{22}}=0$. Finally, the three relations $\underline{N} \cdot \underline{x_{22}}=0, \underline{x_{1}} \cdot \underline{x_{22}}=0, \underline{x_{2}} \cdot \underline{x_{22}}=0$ imply that $\underline{x_{22}}=0$ which shows that the 
parameterization is same as that of the form ruled surface. This ends the proof of the fact that the surface considered is a ruled surface.

Since, here the generators are $u^{1}=$ const., and $\underline{N}_{2}=0$, it follows that $\underline{N}$ is constant along the generator so that the tangent is constant also. Hence, proof of the theorem is complete.

It can be shown by making a suitable example that the statement of the theorem do not hold if $\mathrm{S}$ has planar points.

Sometimes ruled surfaces termed developable surfaces. It is generated by just a single parameter family of planes. Thus there is two important points: (i) tangent plane to a developable surface contains only one parameter, and (ii) if the ruled surface is developable, the tangent planes at all points of any generator must be same.

Theorem 3.2: A surface $S$ has curvature zero if and only if it is developable.

Proof: For particular developable surfaces like cylinder and cone, it is clear that curvature is zero, and if these be excluded, the equation of developable surface can be written $\underline{X}=\underline{x}(s)+\lambda \underline{t}$, from which is possible to show that $M=\underline{N} \cdot \underline{X}_{12}=0$, $N=\underline{X}_{22}=0$ so that curvature $K=\frac{L N-M^{2}}{E G-F^{2}}=0$. Here $E, G, G, L, M, N$ are the fundamental coefficients. Hence the theorem is true.

Theorem 3.3: If tangent plane of a ruled surface $S$ is fixed along the generatos, then $K=0$ on $S$.

Proof: The hypothesis of the fixed tangent plane yields $\underline{N}_{2}=0$, and by the Weingarten transformation, one has $\underline{N}_{2}=-l \underline{x_{2}}$. Hence $\underline{x}_{2}$ is a principal vector with the corresponding principal curvature $k_{1}=0$. Since $K=k_{1} k_{2}$, we get $K=0$.

Theorem 3.4: If a part $S$ of a surface is of curvature zero, then $S$ is isometric with a plane.

Proof: Let two surfaces $S$ and $\bar{S}$ with same constant curvature $K$ be mapped isometrically so that $p \in S$ and $\bar{p} \in \bar{s}$ correspond the same point. Then $d s^{2}=\left(d u^{1}\right)^{2}+f^{2}\left(d u^{2}\right)^{2} \cdots(*)$, where $\mathrm{f}$ is some function of $u^{1}, u^{2}$, and also $K=-f_{11} / f$. If $K=0$, it can be shown that $f=1$ so that $\left(^{*}\right)$ can be written as $d s^{2}=\left(d u^{1}\right)^{2}+\left(d u^{2}\right)^{2}$ which is the metric of the plane. Hence $S$ is isometric with a plane.

The complete geometric structure of the surface of curvature zero can be obtained due to the discussion of the following paragraph.

Let $p$ be any point of the surface $S$ which is of curvature zero. The first case that if $p$ is a planar point. In this situation if a whole nbd. of $p$ consists of planar points, then 
this nbd. belongs to a plane (by proposition 2.2). ${ }^{(1)}$ Thus $p$ belongs to an open $n b d$. of $S$ that lies in a plane.

If $p$ has no such nbd., $p$ is the limit of a sequence of nonplanar points, since every nbd. of $\mathrm{p}$ has nonplanar points, ${ }^{(2)}$ then certainly $p$ belongs to an open nbd. of $S$ that lies in a plane or, in a cylinder or, in a cone.

Now let $p$ be a nonplanar point. Then by theorem 3.3 we have, for example, $k_{1}=0, k_{2}>0$, which allows us to use a parameterization (*) in section 2 at $p$ whose parametric lines are the lines of curvature, and such that $\underline{v}^{2}=1, y^{\prime 2}=1 \quad\left(u^{1}\right.$ is the arc length on $\left.u^{2}=0\right)$. This implies that $b_{12}$ vanishes and that $\underline{v}$ and $\underline{y}$ are orthogonal $\left(g_{11}=0\right)$. It follows that $\underline{v^{\prime}}=\alpha \underline{y}+\beta \underline{v}$ whence, by a scalar multiplication with $\underline{v}$, we deduce that $\beta=0$. That is, $\underline{v^{\prime}}=\bar{\alpha} \underline{y^{\prime}}, \alpha=\underline{y^{\prime}} \underline{v}^{\prime}$. Now, if $\alpha=0$ on an open $n b d$. of $p$, we have $\underline{v}=$ const, and ${ }^{(3)}$ this $n b d$. belongs to a cylinder which is by definition a ruled surface with parallel generators.

If $\alpha(p)=0$ and every nbd of $\mathrm{p}$ has points with a nonvanishing $\alpha, p$ is the limit of a sequence of points with nonvanishing $\alpha$ and we shall again have case ${ }^{(2)}$.

Furthermore, assume that $\alpha(p) \neq 0$, then this holds on a whole $n b d$. of $p$, and let us look for a curve that is tangent to the straight lines containing the generators of $S$. Such a curve must have an equation of the form $\underline{z}=\underline{y}\left(u^{1}\right)+\lambda\left(u^{1}\right) \underline{v}\left(u^{1}\right)$ with the tangent vector $\underline{y}+\lambda^{\prime} \underline{v}+\lambda \underline{v^{\prime}}$. This is collinear to $\underline{v}$ if and only if $\lambda=-\alpha$, where $\alpha$ is given as above. Then if the point $\underline{z}$ with $\lambda=-\alpha$ is a fixed point i.e., $\underline{z}=0$ on a whole $n b d$. of $p$. ${ }^{(4)}$ Then this $n b d$. belongs to a cone, and if $\underline{z}(p) \neq 0$, then clearly ${ }^{(5)}$ the $n b d$. of $p$ lies on a surface generated by the tangents of some space curve.

\section{REFERENCES}

Castro, I. and C. R. Montealegre. 2004. A family of surfaces with constant curvatures in Euclidean four space, Soochow J. Mathematics 30(3): 293-301.

Hossain, M. A. 2010. Structures of surfaces with non-zero constant curvature. Ganit: Journal of Bangladesh Mathematical Society (submitted).

Kenmotsu, K. 1983. Minimal surfaces with constant curvature in 4-dimensional space forms. Proceedings of the AMS 89(1): 133-138.

Kreyszig, E. 1991. Differential geometry, Dover publication Inc., Mineola, New York.

Lopez, R. 2001. Cyclic surfaces of constant gauss curvature. Houston Journal of Mathematics 27(4): 799-805.

Pressley, A. 2001. Elementary differential Geometry, Springer-Verlag, London. 